\title{
Experimental Organism Malignant Astrocytoma
}

National Cancer Institute

\section{Source}

National Cancer Institute. Experimental Organism Malignant Astrocytoma. NCI

Thesaurus. Code C119574.

A malignant neoplasm of the brain or spinal cord originating from astrocytes. 